\title{
Some Currently Neglected Aspects of Cholinergic Function
}

\author{
Victor P. Whittaker
}

Received: 6 July 2009 / Accepted: 20 July 2009 /Published online: 29 September 2009

(C) The Author(s) 2009. This article is published with open access at Springerlink.com

\begin{abstract}
At the request of the organizers of the XIII International Symposium on Cholinergic Mechanisms, I have selected for review three topics not well represented at the meeting: the synthesis, storage, and release of acetylcholine at cholinergic nerve endings; cholinergic-specific antigens; and neuropeptides as cholinergic co-transmitters. The first topic is illustrated by work with two model systems, the electromotor nerve terminals of the electric ray and the myenteric plexus of the guinea-pig ileum, the second by work with Chol-1, a group of cholinergic-specific gangliosides sialylated on their N-acetylgalactosamine residues, and the third by vasoactive intestinal polypeptide which is a co-transmitter at $70 \%$ of cholinergic nerve endings in brain and is also present in both of the model cholinergic systems.
\end{abstract}

Keywords Cholinergic transmission - Cholinergic-specific antigens $\cdot$ Chol- $1 \cdot$ Neuropeptides as cholinergic co-transmitters · VIP · Model cholinergic systems . Electromotor synapses of the electric ray, Torpedo . Myenteric plexus

Proceedings of the XIII International Symposium on Cholinergic Mechanisms

V. P. Whittaker Wolfson College, Cambridge, UK

V. P. Whittaker $(\bowtie)$

54 Gough Way,

Cambridge CB3 9LN, UK

e-mail: whittaker54@talktalk.net

V. P. Whittaker

Max Planck Institute for Biophysical Chemistry,

Göttingen, Germany

\author{
Abbreviations \\ ACh acetylcholine \\ ATP adenosine triphosphate \\ ATPase adenosine triphosphatase \\ ChAT choline acetyltransferase \\ DOG deoxyglucose \\ LDH lactate dehydrogenase \\ MPLM myenteric plexus-longitudinal muscle of the \\ guinea-pig ileum \\ $\mathrm{T}$ transporter \\ VPG vesicular proteoglycan
}

I have been asked by the organizers of the XIII International Symposium on Cholinergic Mechanisms to review topics which are not well, or not at all represented in the program of this meeting, yet present departure points for profitable further work. I have selected three: the synthesis, storage and release of acetylcholine (ACh) at cholinergic nerve terminals; cholinergic-specific antigens; and neuropeptides as cholinergic co-transmitters. Examples will be taken mainly from the work of my own group, much of which has been done with two model systems: the electromotor system of the electric ray, Torpedo, and the superfused myenteric plexus-longitudinal muscle (MPLM) preparation of the guinea-pig ileum. The profusely innervated electrocytes of the electric organ are derived from myocytes: as in muscle the innervation is cholinergic but much more profuse, so that the ACh content of the electric organ is up to 1,000 times that of muscle and 100 times that of brain; MPLM has 100 times more ACh than muscle and 10 times more than brain. Synaptic vesicles can be isolated directly from both tissues; those of electromotor terminals are larger than those of brain or muscle $-90 \mathrm{~nm}$ in diameter versus $50 \mathrm{~nm}$ - but those of MPLM are of normal size. The 
VPG (x20) 200

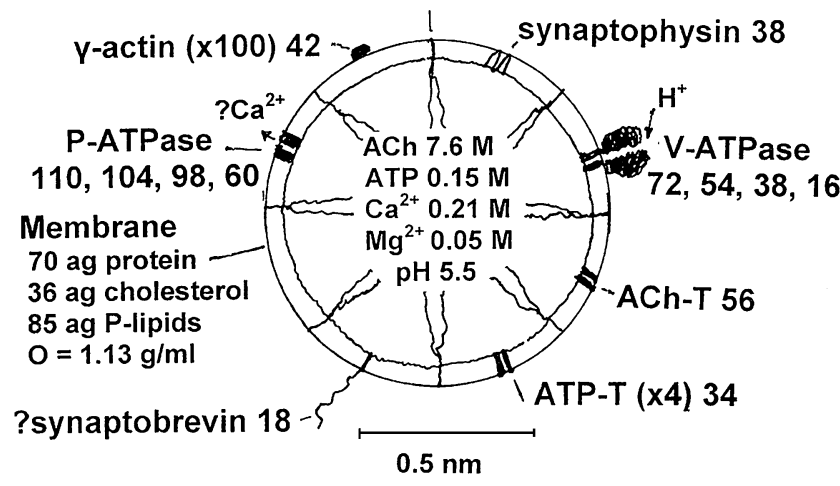

Figure 1 Composition of the reserve $\left(\mathrm{V}_{1}\right)$ synaptic vesicle from the cholinergic electromotor synapse. Number of copies of membrane constituents, if above three and where known, are given in parentheses. For abbreviations, see text

larger size of electromotor vesicles facilitates their subfractionation and biophysical measurements.

\section{Synthesis, Storage, and Release of Acetylcholine}

Before reviewing the results obtained with these preparations, those with brain synaptic vesicles should be briefly mentioned. Nerve terminals take up about $3 \%$ of cortical brain mass, so the discovery (Gray and Whittaker 1960, 1962) that they could be isolated as sealed structures to which we later gave the name synaptosomes provided us, inter alia, with a preparation enriched over 30 -fold with synaptic vesicles. These we could isolate from them in a high state of purity (Whittaker and Sheridan 1965). By labeling our preparations with known amounts of vesiclesized polystyrene beads, we were able to determine the average number of ACh molecules per vesicle as 300. Since cholinergic endings comprise only about $3 \%$ of the total, the molecular ACh content of cholinergic vesicles must be about 6,000 , close to the best estimates of the size of the quantum; this figure is also consistent with a synaptic vesicle filled with an iso-osmotic ACh solution. Using a simplified version of a purification technique developed in my lab (Nagy et al. 1976) and using techniques not available to us, Jahn and coworkers (Takamori et al. 2006) have been able to construct what amounts to a molecular model of the synaptic vesicle. However, this is a model, not of the cholinergic vesicle, but of the predominant kinds, those from glutamergic and gabaergic terminals, which, as we have seen, greatly outnumber cholinergic ones.

The cholinergic electromotor vesicle (Fig. 1) is somewhat simpler than Jahn's model. It contains seven known proteins or groups of protein subunits: the vesicular proteoglycan (VPG) whose negatively charged glycan moieties fill the vesicle core; the $\mathrm{ACh}$ and adenosine triphosphate (ATP) transporters (ACh-T, ATP-T); the vesicular (V-type) proton-translocating adenosine triphosphatase (V-ATPase), which provides, via proton exchange, the driving force for ACh uptake, and indirectly, for that of ATP; the P-type ATPase (P-ATPase) responsible for regulating the $\mathrm{Ca}$ and $\mathrm{Mg}$ content of the vesicle; synaptophysin, possibly an ion channel; and $\gamma$-actin, possibly a link to the cytoskeleton, permitting vesicle movement. In addition, very likely though not positively identified, there is an eighth protein — synaptobrevin — which is present in brain
Figure 2 Cartoon representing the three pools of synaptic vesicles in the cholinergic nerve terminal, their maturation, mobilization, recycling, recovery, and eventual demobilization. For explanation and abbreviations, see text

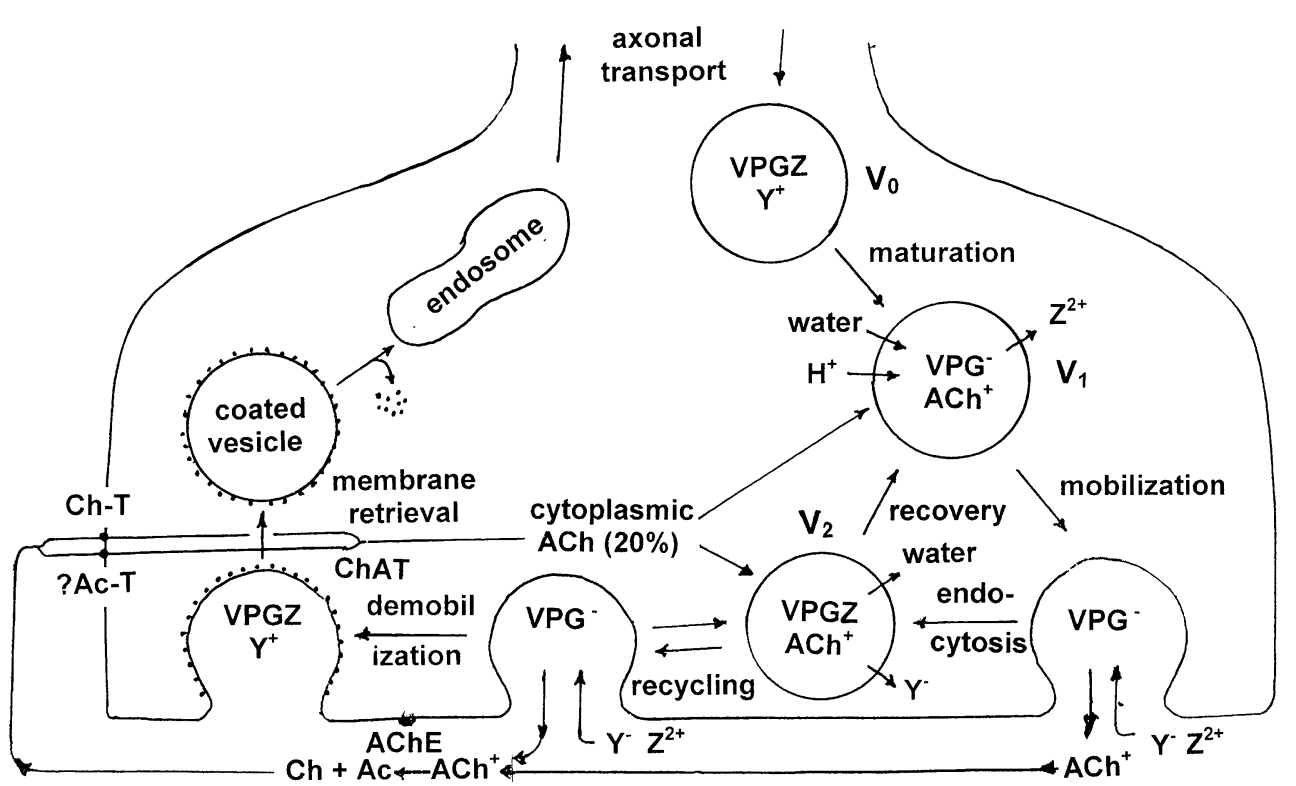


Table 1 Electromotor synaptic vesicles are heterogeneous in size, density, and function

Whittaker (1990)

\begin{tabular}{|c|c|c|c|}
\hline Pool & $\mathrm{V}_{0}$ & $\mathrm{~V}_{1}$ & $\mathrm{~V}_{2}$ \\
\hline Functional state & Axonal & Reserve & Recycling \\
\hline ACh molecules per vesicle & 200 & 246,000 & 79,000 \\
\hline Inorganic ions & High & Low & High \\
\hline Free water $\left(\% \mathrm{~V}_{1}\right)$ & 65 & 65 & 42 \\
\hline Diameter (nm) & 90 & 90 & 70 \\
\hline Density (g/ml) & 1.050 & 1.056 & 1.062 \\
\hline ATPase (nmol/h/100 U synaptophysin) & 240 & 690 & $\mathrm{n} / \mathrm{d}$ \\
\hline
\end{tabular}

vesicles and acts as a 'hook', attaching the vesicle to the cytoplasmic surface of the plasma membrane, a necessary preliminary to vesicle 'docking' and exocytosis.

VPG can be detected immunohistochemically or by ${ }^{35} \mathrm{~S}$ labeling of its sulfate groups. Using these markers we have shown that the vesicles are formed in the cell body, travel down the axon by fast axonal transport, and form a pool of newly arrived vesicles of low ACh content (Fig. 2). This pool is designated the $\mathrm{V}_{0}$ pool. Perhaps by activating their ATPases these vesicles undergo maturation; they lose inorganic ions and acquire ACh and ATP. They enter the reserve or $V_{1}$ pool. On stimulation, a proportion of $V_{1}$ vesicles is mobilized and undergoes exo- and endocytosis. The glycan moieties of VPG may act like an ion-exchange resin; during exocytosis $\mathrm{ACh}^{+}$is exchanged for inorganic ions from the extracellular fluid. The bivalent cations block the negatively charged sulfate groups of the VPG. Recovered vesicles rapidly exchange $\mathrm{ACh}^{+}$for $\mathrm{Na}^{+}$and $\mathrm{K}^{+}$ions and, if stimulation continues, undergo repeated exo- and endocytosis. These recycling vesicles constitute the $\mathrm{V}_{2}$ pool. During an ensuing period of rest, bivalent ions that have blocked the negative charges of VPG ate exchanged via the P-ATPase for more ACh; the resultant increase in the free solute load causes water to enter and the vesicles to swell and become less dense. This recovery process enables the recycled vesicles to rejoin the reserve pool. These changes in ionic composition and accompanying changes in water content

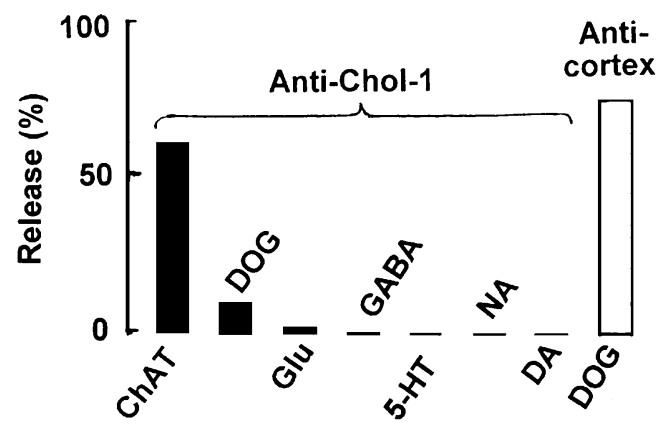

Figure 3 The selective complement lysis of the cholinergic subpopulation of brain cortical synaptosomes after sensitization by an antiChol-1 anti-serum. Radio-labeled transmitter-specific cytoplasmic markers: Glu glutamic, GABA $\gamma$-aminobutyric acids, 5-HT serotonin, $N A$ noradrenaline, $D A$ dopamine. For other abbreviations, see text cause differences in the sizes and densities of the three vesicle pools which make their physical separation possible (Table 1).

After an unknown number of cycles of exo- and endocytosis, vesicles become demobilized and their membranes are salvaged via the endosomal system for reprocessing. In electromotor terminals, coated vesicles are rarely seen, but endosome-like membranes and depleted vesicle numbers are seen after prolonged stimulation. The lipid and protein compositions of the plasma and vesicle membranes are known to remain distinct (Whittaker 1966); this shows that the retrieval of immobilized vesicle membrane is efficient and conservative. The trigger for demobilization is unknown but it may be the loss of glycan residues from VPG.

Released ACh, after combining with its receptors, is rapidly hydrolysed to acetate and choline and salvaged by the presynaptic terminal. Choline is reacetylated by the cytoplasmic enzyme choline acetyltransferase (ChAT), providing a pool of cytoplasmic ACh. This is the source of vesicular ACh. The size of the cytoplasmic ACh pool is 20-25\% (Weiler et al. 1982). Marked differences in the specificities of ChAT and vesicular ACh-T allow the cytoplasmic and vesicular pools to be differentially labeled with choline analogs and compared with the labeled transmitter released on stimulation. Such experiments show that, in the resting synapse, there is little exchange between

Table 2 Structure of Chol-1 Gangliosides

$$
\begin{array}{ccc}
\text { Cer-glc-gal } & \multicolumn{3}{c}{\text {-galNAc-gal }} \\
\text { I } & \text { I } & \text { I } \\
\mathrm{S}(\mathrm{S}) & \mathrm{S} & (\mathrm{S})
\end{array}
$$

Chol-1 gal $_{1}$ galNAc gal $_{2}$ Systematic nomenclature

$\begin{array}{lllll}\alpha \mathrm{a} & \mathrm{S} & \mathrm{S} & \mathrm{S} & \text { GT1a } \alpha \\ \alpha \mathrm{b} & \mathrm{S}-\mathrm{S} & \mathrm{S} & \mathrm{S} & \text { GQ1b } \alpha \\ \beta \mathrm{a} & \mathrm{S} & \mathrm{S} & - & \text { GD1a } \alpha \\ \beta \mathrm{b} & \mathrm{S}-\mathrm{S} & \mathrm{S} & - & \text { GT1b } \alpha \\ \gamma & - & \mathrm{S} & - & \text { GM1 } \alpha\end{array}$

Abbreviations cer ceramide $\mathrm{C}_{18}$ with some $\mathrm{C}_{20}$, glc glucosyl, $\mathrm{gal}_{1}$ internal galactosyl, galNAc N-acetylgalactosamine, gal ${ }_{2}$ terminal galactosyl, $S$ sialyl (Whittaker and Kelic 1995) 
Table 3 Copurification of VIP with cholinergic rat brain cortical synaptosomes affinity-purified using sheep anti-Chol-1 anti-serum and immobilized rat anti-sheep IgG

\begin{tabular}{lcl}
\hline Component & Purification factor & Yield (\%) \\
\hline ACh & 19 & - \\
Chat & 15 & 3.3 \\
VIP & 8 & 4.0 \\
LDH & 1 & - \\
\hline
\end{tabular}

Agoston et al. (1988a)

the cytoplasmic and $\mathrm{V}_{1}$ pools, and that the source of released transmitter is the recycling vesicular and not the cytoplasmic or reserve vesicular pools (Whittaker and Luqmani 1980; Luqmani et al. 1980). The cartoon (Fig. 2) summarizes the various processes involved.

Work with MPLM (Agoston et al. 1985) has shown that in this tissue too $V_{1}$ and $V_{2}$ vesicle pools exist but, due to the small size of the vesicles, they differ less in density and so are less well separated by density gradient centrifuging that the corresponding two pools of electromotor vesicles.

\section{Cholinergic-Specific Antigens}

We prepared presynaptic plasma membranes from Torpedo electric organs and injected them into sheep. The resulting anti-serum, after purification by adsorption, proved to be specific for cholinergic neurones in the mammalian brain. Figure 3 shows one of a number of ways in which this was demonstrated (data plotted from Richardson 1983). Synaptosomes, like cells, can be sensitized to lysis by complement by pretreating them with an anti-serum which recognizes an antigen on the plasma membrane. A mammalian brain cortical synaptosome preparation was treated with our putative cholinergic-specific anti-serum, complement applied and the release of various transmitter-specific cytoplasmic markers measured. For cholinergic synaptosomes the marker was ChAT; glutamergic, gabaergic, serotonergic, noradrenergic, and dopaminergic synaptosomes were loaded with the appropriate radio-labeled cytoplasmic transmitter precursor. Radio-labeled deoxyglucose (DOG) served as a non-specific cytoplasmic marker and an anti-cortex antiserum as a non-specific sensitizer.

It will be seen that of all the transmitter-specific cytoplasmic markers, only ChAT is released when the preparation is sensitized by the putative cholinergic-specific anti-serum. Significantly, this release is accompanied by the release of about $5 \%$ of DOG, about the proportion of cholinergic synaptosomes present in the preparation. By contrast, all the synaptosomes are sensitized by the anticortex anti-serum as shown by the extensive release of DOG in this case.

Work by Japanese colleagues (for refs see Whittaker and Kelic 1995) has identified the antigen recognized by our anti-serum, which we called Chol-1, as a hitherto unknown family of brain gangliosides, characterized by sialylation of the $\mathrm{N}$-acetylgalactosamine residue (Table 2). Although these gangliosides account for only $0.1 \%$ of total brain gangliosides, when allowance is made for the small
Table 4 Co-localization of neuropeptides in cholinergic neurons

For references see Whittaker

(1992)

CGRP calcitonin-gene-related peptide, GRP gastrin-releasing peptide, $S P$ substance $\mathrm{P}, V I P$ vasoactive intestinal polypeptide

\begin{tabular}{lll}
\hline Function/localization & Species & Neuropeptide \\
\hline $\begin{array}{l}\text { Motor/secretomotor } \\
\text { Parasympathetic ganglia }\end{array}$ & Cat \\
Myenteric plexus- longitudinal muscle & Guinea pig & VIP \\
& & VIP, SP, \\
& & Somatostatin \\
Hypoglossal \& facial nuclei, & Guinea pig & Cholecystokinin \\
Nucleus ambiguus & & Neuropeptide Y \\
Vasomotor & & CGRP \\
Cerebral cortex & Rat & \\
Electromotor & & VIP \\
Electromotor neurons & Torpedo spp & VIP, GRP \\
Sensory & & Enkephalin \\
Superior olive & Guinea pig & \\
Activation system & & SP \\
Lateral dorsal segmental & Rat & Galantine \\
Higher brain function & & \\
Medial septal neurons, diagonal band, & Rat & \\
Hippocampal afferents & & \\
\hline
\end{tabular}


contribution that cholinergic endings make to total brain mass, the Chol-1 gangliosides could be a major constituent of the gangliosides of the cholinergic presynaptic terminal membrane.

\section{Neuropeptides as Cholinergic Co-transmitters}

The anti-Chol-1 anti-serum has been applied to the isolation of the cholinergic subfraction of brain cortical synaptosomes (Agoston et al. 1988a). After treatment with the antiserum, the synaptosomes were passed through a column of an immobilized high-titre monoclonal mouse anti-sheep $\mathrm{IgG}$ antibody. The non-cholinergic synaptosomes had not bound anti-serum, so were not recognized by the column and could be washed through, leaving the cholinergic synaptosomes attached to the column. The enrichment of $\mathrm{ACh}$ and ChAT corresponded to an expected ca 5\% abundance of cholinergic synaptosomes in the whole population (Table 3). By contrast, lactate dehydrogenase (LDH), a general cytoplasmic marker, was not enriched.

Interestingly, the neuropeptide vasoactive intestinal polypeptide (VIP) is also enriched, but only to half the extent of the cholinergic marker. Long suspected from immunocytochemical observations to be associated with cholinergic neurones, these results suggest that $50 \%$ of cholinergic synaptosomes contain VIP. However, the yield of VIP is higher than that of ChAT, suggesting that $70 \%$ of VIP-containing endings also contain ChAT, i.e., are also cholinergic.

VIP is also found in association with cholinergic neurones in the peripheral nervous system, the electromotor system being no exception (Agoston et al. 1989). It is packaged in larger 'throw away' vesicles which, unlike synaptic vesicles, cannot be refilled in the periphery and are thus entirely dependent on axonal transport for replenishment. This is well illustrated by some work we did with the MPLM preparation (Agoston et al. 1988b). The release of VIP on stimulation was quickly blocked by colchicine, an axonal transport blocker, while that of acetylcholine was unaffected over a similar period. By contrast, hemicholinium, which blocks the uptake of choline by nerve terminals and so inhibits the synthesis of $\mathrm{ACh}$ and its uptake by recycling vesicles, quickly brought its release to an end while leaving that of VIP unaffected.

Table 4 shows that VIP, while it is the peptide most often associated with cholinergic neurones, is by no means the only ones to be so. What is the function of these potential co-transmitters? The difference in the dynamics of release of neuropeptides and small molecular mass transmitters may suggest that the neuropeptides are a kind of 'memory' of previous neuronal activity. But this is a vast field for further work.
Those interested in exploring the topics raised in this article may consult Whittaker (1992) and Whittaker and Kelic (1995) for references and further results.

Open Access This article is distributed under the terms of the Creative Commons Attribution Noncommercial License which permits any noncommercial use, distribution, and reproduction in any medium, provided the original author(s) and source are credited.

\section{References}

Agoston, D. V., Kosh, J. W., Lisziewicz, J., \& Whittaker, V. P. (1985). Separation of recycling and reserve synaptic vesicles from cholinergic nerve terminals of the myenteric plexus of guineapig ileum. J Neurochem, 44, 299-305.

Agoston, D. V., Borroni, E., \& Richardson, P. J. (1988a). Cholinergic surface antigen Chol-1 is present in a subclass of VIP-containing rat cortical synaptosomes. $J$ Neurochem, 50, 1659-1662.

Agoston, D. V., Conlon, J. M., \& Whittaker, V. P. (1988b). Selective depletion of the acetylcholine and vasoactive intestinal polypeptide of the guinea-pig myenteric plexus by differential mobilization of distinct transmitter pools. Exp Brain Res, 72, 535-542.

Agoston, D. V., Dowe, G. H. C., \& Whittaker, V. P. (1989). Isolation and characterization of secretory granules storing a vasoactive intestinal polypeptide-like peptide in Torpedo cholinergic electromotor neurones. J Neurochem, 52, 1729-1740.

Gray, E. G., \& Whittaker, V. P. (1960). The isolation of synaptic vesicles from the central nervous system. J Physiol, 153, 35-37P.

Gray, E. G., \& Whittaker, V. P. (1962). The isolation of nerve endings from brain: An electron-microscopic study of cell fragments derived by homogenization and centrifugation. $J$ Anat (Lond), 96, 79-88.

Luqmani, Y. A., Sudlow, G., \& Whittaker, V. P. (1980). Homocholine and acetylhomocholine: False transmitters in the cholinergic electromotor system of Torpedo. Neuroscience, 5, 153-160.

Nagy, A., Baker, R. R., Morris, S. J., \& Whittaker, V. P. (1976). The preparation and characterization of synaptic vesicles of high purity. Brain Res, 109, 285-309.

Richardson, P. J. (1983). Presynaptic distribution of the cholinergic-specific antigen and 5 'nucleotidase in rat brain as determined by complement mediated release of transmitter. $J$ Neurochem, 41, 640-648.

Takamori, S., Holt, M., Stenius, K., Lemke, E. A., et al. (2006). Molecular anatomy of a trafficking organelle. Cell, 127, 831846.

Weiler, M., Roed, I. S., \& Whittaker, V. P. (1982). The kinetics of acetylcholine turnover in a resting cholinergic nerve terminal and the magnitude of the cytoplasmic compartment. J Neurochem, $38,1167-1191$.

Whittaker, V. P. (1966). Some properties of synaptic membranes isolated from the central nervous system. Ann N Y Acad Sci, 137, 982-988.

Whittaker, V. P. (1990). Cholinergic synaptic vesicles are metabolically and biophysically heterogeneous even in resting terminals. Brain Res, 511, 113-121.

Whittaker, V. P. (1992). The cholinergic neuron and its target. Boston: Birkhäuser.

Whittaker, V. P., \& Sheridan, M. N. (1965). The morphology and acetylcholine content of isolated cerebral cortical synaptic vesicles. J Neurochem, 12, 363-372.

Whittaker, V. P., \& Luqmani, Y. A. (1980). False transmitters in the cholinergic system: Implications for the vesicle theory of transmitter storage and release. Gen Pharmacol, 11, 7-14.

Whittaker, V. P., \& Kelic, S. (1995). Cholinergic-specific glycoconjugates. Neurochem Res, 20, 1377-1387. 